\title{
Urinary angiotensinogen as a surrogate marker predicting the antiproteinuric effects of angiotensin receptor blockers in patients with overt proteinuria: a multicenter prospective study
}

Junseok Jeon ${ }^{1+}$, Do Hee Kim ${ }^{2 \dagger}$, Hye Ryoun Jang ${ }^{*}$, Jung Eun Lee ${ }^{1}$, Wooseong Huh', Hye-Young Kim², Dae Joong Kim ${ }^{1}$ and Yoon-Goo Kim ${ }^{1}$

\begin{abstract}
Background: Although urinary angiotensinogen (AGT) and renin reflect intrarenal renin-angiotensin system activity and are enhanced in proteinuric chronic kidney disease, the clinical value of urinary AGT and renin levels during antiproteinuric treatment has yet to be determined. We investigated the clinical usefulness of initial urinary AGT or renin to determine the antiproteinuric effects of angiotensin receptor blockers (ARBs).

Methods: This multicenter, prospective, single-arm study included 205 patients with overt proteinuria (urinary protein/creatinine ratio [UPCR] $\geq 1 \mathrm{mg} / \mathrm{mg}$ ) enrolled between April 2009 and December 2011. All patients were treated with valsartan. The urinary AGT/creatinine ratio (UAGT/Cr) was measured at the baseline and 24 weeks, and the renin/creatinine ratio ( $\mathrm{uR} / \mathrm{Cr}$ ) was measured at the baseline. Fifty-six patients were followed-up for 5 years.
\end{abstract}

Results: The mean age was 47.6 years and $51.2 \%$ were male. The mean uPCR was $2.32 \mathrm{mg} / \mathrm{mg}$ and the mean eGFR was $63.2 \mathrm{~mL} / \mathrm{min} / 1.73 \mathrm{~m}^{2}$. Natural logarithms (In) (UAGT/Cr), In(UR/Cr), and diabetes mellitus were associated with proteinuria decrement (decrease in UPCR $\geq 1 \mathrm{mg} / \mathrm{mg}$ ). Ln(UAGT/Cr) was an independent predictor for proteinuria decrement (OR 1.372, 95\% Cl, 1.068-1.762, $P=0.013$ ). Among the 56 patients followed-up for 5 years, $\Delta \ln (\mathrm{UAGT} / \mathrm{Cr}$ ) at 24 weeks was an independent predictor for $\mathrm{UPCR}<1 \mathrm{mg} / \mathrm{mg}$ at 5 years (OR $0.379,95 \% \mathrm{Cl}, 0.20-0.715, P=0.003$ ).

Conclusions: Our study demonstrates the potential role of both baseline urinary AGT and changes in urinary AGT during the initial 24 weeks as surrogate markers predicting the antiproteinuric effects of ARBs in patients with overt proteinuria.

Keywords: Urinary angiotensinogen, Urinary renin, Intrarenal renin angiotensin system, Angiotensin receptor blocker, Proteinuria

\footnotetext{
* Correspondence: shinehr@gmail.com

${ }^{\dagger}$ Junseok Jeon and Do Hee Kim contributed equally to this work.

'Division of Nephrology, Department of Medicine, Samsung Medical Center,

Sungkyunkwan University School of Medicine, 81 Irwon-ro, Gangnam-gu,

Seoul 06351, Republic of Korea

Full list of author information is available at the end of the article
}

(C) The Author(s). 2020 Open Access This article is licensed under a Creative Commons Attribution 4.0 International License, which permits use, sharing, adaptation, distribution and reproduction in any medium or format, as long as you give appropriate credit to the original author(s) and the source, provide a link to the Creative Commons licence, and indicate if changes were made. The images or other third party material in this article are included in the article's Creative Commons licence, unless indicated otherwise in a credit line to the material. If material is not included in the article's Creative Commons licence and your intended use is not permitted by statutory regulation or exceeds the permitted use, you will need to obtain permission directly from the copyright holder. To view a copy of this licence, visit http://creativecommons.org/licenses/by/4.0/ The Creative Commons Public Domain Dedication waiver (http://creativecommons.org/publicdomain/zero/1.0/) applies to the data made available in this article, unless otherwise stated in a credit line to the data. 


\section{Background}

Inhibition of the renin-angiotensin system (RAS) is a cornerstone of managing chronic kidney disease (CKD) patients with proteinuria including diabetic nephropathy and glomerulonephritis. Previous studies have shown that angiotensin-converting enzyme inhibitors (ACEi) or angiotensin II receptor blockers (ARBs) that inhibit RAS mitigate the progression of $\mathrm{CKD}$ by reducing proteinuria beyond their blood pressure-lowering effects [1, 2]. Regulation of local RAS is independent of systemic RAS in various tissues, including the kidneys, and intrarenal RAS has all RAS components in contrast to other organs [3]. Activated intrarenal RAS plays an important role in the pathogenesis of hypertension and CKD progression $[4,5]$. Urinary angiotensinogen (AGT) and renin reflect the activity of the intrarenal RAS [6-8]. Since the enzyme-linked immunosorbent assay (ELISA) system measuring AGT was developed, several clinical studies have demonstrated that urinary AGT excretion increases in patients with hypertension [9], CKD [7, 10], or diabetes mellitus (DM) [11] regardless of plasma AGT levels.

The degree of proteinuria is strongly associated with CKD progression and cardiovascular disease [12]. Prediction or enhancement of the antiproteinuric effects of RAS inhibitors are important issues in the management of CKD [13, 14]. Previous studies have reported that urinary AGT excretion is positively correlated with the degree of proteinuria $[7,15]$ and is decreased by RAS inhibitors $[9,16]$. Increased urinary AGT excretion may proceed the aggravation of albuminuria $[11,17]$. On the other hand, urinary renin excretion may also be a potential marker for intrarenal RAS activity or a predictor of the antiproteinuric effects of RAS inhibitors [8, 18]. However, few studies have investigated the relationship between the degree of urinary AGT or renin excretion and the antiproteinuric effects of RAS inhibitors $[8,16]$.

In this prospective study, we aimed to investigate the clinical relevance of urinary AGT or renin in the treatment of proteinuria by analyzing the association between urinary AGT or renin excretion and the antiproteinuric effects of RAS inhibitors.

\section{Methods}

\section{Study design and patient selection}

This multicenter, prospective, single-arm study was conducted at eleven tertiary hospitals in South Korea. A total of 323 adult patients under the age of 70 with overt proteinuria (urinary protein to creatinine ratio $[\mathrm{UPCR}] \geq 1 \mathrm{mg}$ / $\mathrm{mg}$ ) were enrolled among patients who visited the nephrology clinic between April 2009 and December 2011. Blood pressure was relatively well controlled, ranging from 100/60 to $160 / 100 \mathrm{mmHg}$, in all patients. Low-salt diet was thoroughly educated in all patients at the time of enrollment. The exclusion criteria were as follows: an estimated glomerular filtration rate (eGFR) less than $30 \mathrm{~mL} / \mathrm{min} /$ $1.73 \mathrm{~m}^{2}$, the use of non-interruptible drugs that affect proteinuria, patients who received immunosuppressive treatment during the preceding 6 months, severe proteinuria with $\mathrm{uPCR}>10 \mathrm{mg} / \mathrm{mg}$, serum albumin $<2.5 \mathrm{~g} / \mathrm{dL}$ or refractory edema, uncontrolled DM with $\mathrm{HbA} 1 \mathrm{C}>9.0 \%$, underlying conditions that may affect intrarenal RAS activity (e.g., renal artery stenosis, primary aldosteronism, or pheochromocytoma), cardiovascular events during the preceding 12 months (e.g., myocardial infarction, unstable angina, coronary artery bypass surgery, percutaneous transluminal coronary angioplasty, cerebrovascular accident, or transient ischemic attack), hypokalemia or hyperkalemia ( $\mathrm{K} \leq 3.5$ or $\geq 5.5 \mathrm{mmol} / \mathrm{L}$, respectively), abnormal liver function (aspartate transaminase or alanine transaminase higher than twice the upper limit of the normal range), allergic reaction to angiotensin-converting enzyme inhibitors (ACEi) or angiotensin II receptor blockers (ARBs), or pregnant or nursing patients. We enrolled patients with $\mathrm{uPCR} \geq 1 \mathrm{mg} / \mathrm{mg}$ and $<10 \mathrm{mg} / \mathrm{mg}$ to maximize the clinical effect of antiproteinuric effect and exclude full-blown nephrotic syndrome patients who are at high risk for rapid progression with RAS inhibitor treatment. Patient with advanced CKD (eGFR $\left.<30 \mathrm{~mL} / \mathrm{min} / 1.73 \mathrm{~m}^{2}\right)$ were excluded considering the relative high risk-benefit ratio of $\mathrm{ACEi} / \mathrm{ARB}$ and potential interference of renal function on urinary AGT or renin.

Study protocol was summarized in Fig. 1. Valsartan was selected based on the extensively studied antiproteinuric effects and the availability of several dose formularies. Patients received only conventional supportive care without immunosuppressive treatment prior to the study period. If patients were already taking $\mathrm{ACEi}$ or $\mathrm{ARB}$ before the screening, the drugs were discontinued for at least 4 weeks. Valsartan treatment was started at the beginning of the study with $80 \mathrm{mg}$ for patients who had not previously taken ACEi or ARB and $160 \mathrm{mg}$ for those who had been on ACEi or ARB before the enrollment. If $\mathrm{UPCR}>1 \mathrm{mg} /$ $\mathrm{mg}$, systolic blood pressure $>160 \mathrm{mmHg}$, or diastolic pressure $>100 \mathrm{mmHg}$ at 4 weeks or 8 weeks, the dose of valsartan was increased to $160 \mathrm{mg}$ at 4 weeks and then 320 $\mathrm{mg}$ at 8 weeks in patients whose initial dose was $80 \mathrm{mg}$. The dose of valsartan was increased to $320 \mathrm{mg}$ at 8 weeks in patients whose initial dose was $160 \mathrm{mg}$.

\section{Sample collection and measurement of intrarenal RAS activity}

Clinical data, including age, sex, underlying comorbidities, and blood pressure, were extracted from electronic medical records. Blood pressures were measured at each clinic visit after a rest period in a chair using an automated blood pressure device. Laboratory data, including hemoglobin, albumin, cholesterol, uric acid, blood urea nitrogen (BUN), serum creatinine $(\mathrm{sCr})$, eGFR, electrolytes, and $\mathrm{uPCR}$, were 


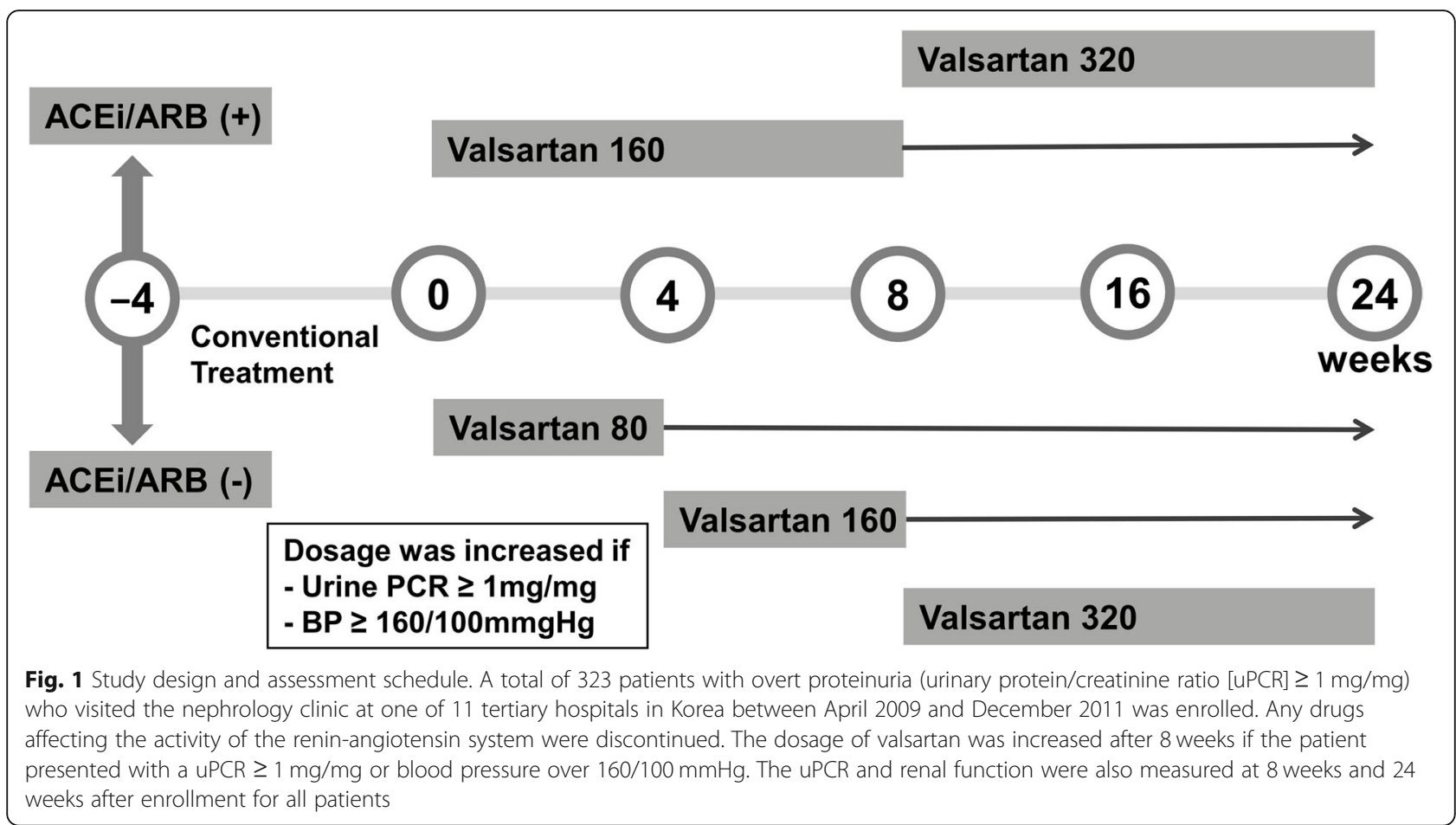

also collected. The eGFR was calculated based on the modified Modification of Diet in Renal Disease using sCr [19]. All urine samples were measured in a central laboratory.

To measure urinary AGT and renin, overnight-fasting early-morning urine samples were collected before starting valsartan treatment. Samples were centrifuged at $2500 \mathrm{rpm}$ for $15 \mathrm{~min}$ at $4{ }^{\circ} \mathrm{C}$, and the supernatants were then stored at $-70^{\circ} \mathrm{C}$. The urine samples were thawed and centrifuged at $13,000 \mathrm{rpm}$ for $2 \mathrm{~min}$ at $4{ }^{\circ} \mathrm{C}$ just prior to use in the enzyme-linked immunosorbent assay (ELISA) for AGT or the radioimmunoassay for renin. Sandwich ELISAs were performed to quantify urinary AGT (uAGT) using mouse monoclonal and rabbit polyclonal antibodies against recombinant human AGT as previously reported [20, 21]. Highly purified human AGT (Calbiochem, Beeston, UK) was used as the standard. The correlation coefficient of the assay was greater than 0.99 , and the detection limit of the ELISA system was $0.9 \mathrm{ng} / \mathrm{mL}$. The urinary renin activity was measured using renin radioimmunoassay beads (TFB, Tokyo, Japan) and a Cobra-II gamma counter (Packard Bioscience, Meriden, CT, USA) as previously reported [8]. Prior to measurement, the urine sample was concentrated 5-fold with an Amicon Ultra-10 centrifugal filter device (Millipore, Cork, Ireland). The detection limit of the enzymatic-kinetic assay was $0.10 \mathrm{ng}$ angiotensin $\mathrm{I} / \mathrm{mL} / \mathrm{h}$. The renin concentration was determined from the angiotensin I-generating activity with $1 \mathrm{ng}$ angiotensin $\mathrm{I} / \mathrm{mL} / \mathrm{h}$ corresponding to $2.6 \mathrm{pg}$ human $\mathrm{renin} / \mathrm{mL}[8,22]$.

To correct for the difference in urinary concentrations, the urinary AGT and renin levels were divided by the urinary creatinine level, resulting in a urinary AGT/creatinine ratio ( $\mathrm{uAGT} / \mathrm{Cr}$ ) and a urinary renin/creatinine ratio $(\mathrm{uR} / \mathrm{Cr})$, respectively. The $\mathrm{uAGT} / \mathrm{Cr}$ and $\mathrm{uR} / \mathrm{Cr}$ levels were then compared after natural logarithmic conversion due to skewed distributions. Delta $\ln (\mathrm{uAGT} / \mathrm{Cr})$ $[\Delta \ln (\mathrm{uAGT} / \mathrm{Cr})]$ was defined as $\ln (\mathrm{uAGT} / \mathrm{Cr})$ at 24 weeks minus the baseline $\ln (\mathrm{uAGT} / \mathrm{Cr})$, and $\triangle \mathrm{uPCR}$ was defined as UPCR at either 24 weeks or 5 years after enrollment minus the baseline uPCR.

\section{Follow-up and outcome measures}

The uPCR was measured at 8 weeks and 24 weeks after enrollment in all patients (Fig. 1). The concentrations of urine protein were measured by a colorimetric assay. Baseline $\ln (\mathrm{uAGT} / \mathrm{Cr})$ and $\ln (\mathrm{uR} / \mathrm{Cr})$, as well as $\Delta \ln (\mathrm{uAGT} / \mathrm{Cr})$, were compared between patients with an uPCR decrement $\geq 1 \mathrm{mg} / \mathrm{mg}$ (decrement group) and patients with an uPCR decrement $<1 \mathrm{mg} / \mathrm{mg}$ (non-decrement group) during valsartan treatment. Furthermore, other possible prognostic factors for proteinuria decrement were analyzed using multivariable logistic regression analysis. Subgroup analyses regarding predictive factors for a uPCR less than $1 \mathrm{mg} / \mathrm{mg}$ and changes in eGFR were conducted for 56 patients who were followed up for 5 years after enrollment.

\section{Statistical analyses}

Continuous variables with a normal distribution are expressed as the mean \pm standard deviation (SD), and variables with a non-normal distribution are expressed as the median with interquartile range (IQR). Group means were 
compared using the $t$-test and medians were compared using the Mann-Whitney test. Serial changes were compared using the paired $t$-test or the Wilcoxon signed-rank test, and repeated measures with analysis of variance (ANOVA), as appropriate. Pearson's correlation analysis was used to test correlations between two continuous variables. Multivariable logistic regression analysis was performed using variables with $P<0.1$ in the univariable models. For all tests, a $P$-value $<0.05$ was considered statistically significant. All statistical analyses were conducted using IBM SPSS software, version 23 (IBM Corporation, Armonk, NY, USA).

\section{Results}

\section{Baseline characteristics of the study population}

After excluding patients who withdrew consent, were lost follow-up, or did not have data for urinary protein/ creatinine ratio at 24 weeks of valsartan treatment, a total of 205 patients were included in the final analyses. The baseline characteristics and follow-up data at 8 weeks and 24 weeks of the 205 patients are summarized in Table 1. The mean age was $47.6 \pm 12.5$ years, and 105 patients (51.2\%) were male. A total of 80 patients (39\%) and 39 patients (19\%) had hypertension and DM, respectively. Baseline median $\ln (\mathrm{uAGT} / \mathrm{Cr})$ and $\ln (\mathrm{uR} /$ $\mathrm{Cr}$ ) were [median (interquartile range, IQR)] 3.49 (2.734.31) and - 1.14 (-2.08-0.62), respectively. Baseline $\ln (\mathrm{uAGT} / \mathrm{Cr})$ and $\ln (\mathrm{uR} / \mathrm{Cr})$ were positively correlated with baseline uPCR $(R=0.455, P<0.001$ and $R=0.317$, $P<0.001$, respectively) and negatively correlated with baseline eGFR $(R=-0.352, P<0.001$ and $R=-0.214$, $P<0.001$, respectively).

\section{Changes in proteinuria, renal function, and urinary AGT excretion during valsartan treatment}

Proteinuria decreased at 8 weeks and 24 weeks from the baseline after starting valsartan. However, there were no significant difference between proteinuria at 8 weeks and at 24 weeks (baseline vs. 8 weeks vs. 24 weeks; mean uPCR, $\mathrm{mg} / \mathrm{mg}: 2.32 \pm 1.43$ vs. $2.06 \pm 1.63$ vs $2.02 \pm 1.93$, $P=0.003)$. The eGFR also decreased slightly at 8 weeks similar to proteinuria (baseline vs. 8 weeks vs. 24 weeks; mean eGFR, $\mathrm{mL} / \mathrm{min} / 1.73 \mathrm{~m}^{2}: 63.2 \pm 28.8$ vs. $61.0 \pm 27.5$ vs. $61.6 \pm 29.7, P=0.003)$. $\mathrm{Ln}(\mathrm{uAGT} / \mathrm{Cr})$ decreased at 24 weeks after starting valsartan [baseline vs. 24 weeks; median $\ln (\mathrm{uAGT} / \mathrm{Cr}), \mu \mathrm{g} / \mathrm{g}: 3.49$ (2.73-4.31) vs. 3.16 (2.174.26), $P=0.005]$.

Table 1 Clinical characteristics of the study population during valsartan treatment

\begin{tabular}{|c|c|c|c|c|c|}
\hline & Baseline & 8 weeks & 24 weeks & $P^{\mathrm{a}}$ & $P^{b}$ \\
\hline Age (years) & $47.6 \pm 12.5$ & & & NA & NA \\
\hline Male & $105(51.2)$ & & & NA & NA \\
\hline \multicolumn{6}{|l|}{ Comorbid disease } \\
\hline Hypertension & $80(39.0)$ & & & NA & NA \\
\hline Diabetes mellitus & $39(19.0)$ & & & NA & NA \\
\hline Mean arterial pressure $(\mathrm{mmHg})$ & $111.6 \pm 12.0$ & $110.9 \pm 15.3$ & $110.4 \pm 12.6$ & 0.510 & 0.142 \\
\hline \multicolumn{6}{|l|}{ Blood sample } \\
\hline BUN (mg/dL) & $20.1 \pm 7.83$ & $20.4 \pm 8.04$ & $21.2 \pm 9.39$ & 0.388 & $<0.001$ \\
\hline Serum creatinine (mg/dL) & $1.27 \pm 0.46$ & $1.30 \pm 0.48$ & $1.32 \pm 0.53$ & 0.001 & $<0.001$ \\
\hline eGFR $\left(\mathrm{mL} / \mathrm{min} / 1.73 \mathrm{~m}^{2}\right)$ & $63.2 \pm 28.8$ & $61.0 \pm 27.5$ & $61.6 \pm 29.7$ & 0.003 & 0.009 \\
\hline Serum albumin (mg/dL) & $4.02 \pm 0.45$ & $4.04 \pm 0.58$ & $4.06 \pm 0.58$ & 0.193 & 0.301 \\
\hline Serum cholesterol (mg/dL) & $181.8 \pm 35.6$ & $180.4 \pm 36.4$ & $178.4 \pm 33.9$ & 0.429 & 0.245 \\
\hline Serum uric acid (mg/dL) & $6.91 \pm 1.86$ & $7.02 \pm 1.88$ & $6.94 \pm 1.77$ & 0.264 & 0.847 \\
\hline \multicolumn{6}{|l|}{ Urine sample } \\
\hline $\mathrm{uPCR}(\mathrm{mg} / \mathrm{mg})$ & $1.85(1.34-2.76)$ & $1.69(0.99-2.57)$ & $1.40(0.81-2.58)$ & $<0.001$ & $<0.001$ \\
\hline $\ln (U A G T / C r)(u g / g)$ & $3.49(2.73-4.31)$ & $3.36(2.21-4.06)$ & $3.16(2.17-4.26)$ & 0.079 & 0.005 \\
\hline $\ln (U R / C r)(p g / g)$ & $-1.14(-2.08-0.62)$ & & & NA & NA \\
\hline $\mathrm{uNa} / \mathrm{Cr}(\mathrm{mmol} / \mathrm{mmol})$ & $11.4(7.2-18.8)$ & $11.0(6.8-17.5)$ & $11.7(8.1-17.3)$ & 0.242 & 0.185 \\
\hline
\end{tabular}

Abbreviations: BUN blood urea nitrogen, eGFR estimated glomerular filtration rate, $N A$ not applicable, $u A G T / C r$ urinary angiotensinogen/creatinine ratio, $u N a / C r$ urinary sodium/creatinine ratio, $u P C R$ urinary protein/creatinine ratio, $u R / C r$ urinary renin/creatinine ratio

Continuous variables with a normal distribution are expressed as the mean \pm standard deviation, and those with non-normal distribution are expressed as the median (interquartile range). Categorical variables are expressed as number (percentage). Variables were compared using the paired $t$-test or the Wilcoxon signedrank test as appropriate

A $P$-value $<0.025$ was considered statistically significant in this table by applying Bonferroni correction method

a $P$ : baseline vs. 8 weeks

${ }^{\mathrm{b}} P$ : baseline vs. 24 weeks 
Baseline urinary RAS activity by proteinuria decrement We compared baseline $\ln (\mathrm{uAGT} / \mathrm{Cr}), \ln (\mathrm{uR} / \mathrm{Cr})$, and $\Delta \ln (\mathrm{uAGT} / \mathrm{Cr})$ between patients with a uPCR decrement $\geq 1 \mathrm{mg} / \mathrm{mg}$ (decrement group) and patients with an uPCR decrement $<1 \mathrm{mg} / \mathrm{mg}$ (non-decrement group) at 24 weeks after starting valsartan (Table 2). Baseline $\ln (\mathrm{uAGT} / \mathrm{Cr})$ was significantly higher in the decrement group compared to the non-decrement group [median (IQR) in the decrement vs. the non-decrement groups: $3.92(3.39-4.59)$ vs. 3.29 (2.53-4.21), $P=0.004]$. Baseline $\ln (\mathrm{uR} / \mathrm{Cr})$ was also significantly higher in the decrement group compared to the non-decrement group [median (IQR) in the decrement vs. the non-decrement groups: $-0.35(-1.96-1.73)$ vs. $-1.25(-2.11-0.47), P=0.047]$. $\mathrm{Ln}(\mathrm{uAGT} / \mathrm{Cr})$ decreased more in the decrement group compared to the non-decrement group at 24 weeks [median (IQR) of $\Delta \ln (\mathrm{uAGT} / \mathrm{Cr})$ in the decrement vs. the non-decrement groups: $-0.76(-2.07-0.80)$ vs. 0.00 (0.79-0.63), $P<0.001$, respectively]. Doses of valsartan or baseline mean arterial pressure between the decrement and non-decrement group were similar. Baseline proteinuria levels were higher in the decrement group than the non-decrement group.

\section{Predictive factors for the antiproteinuric effects of valsartan}

We conducted a logistic regression analysis to identify predictive factors for proteinuria decrement (decrease in $\mathrm{uPCR} \geq 1 \mathrm{mg} / \mathrm{mg}$ at 24 weeks) (Table 3 ). The univariable analysis found $\mathrm{DM}, \ln (\mathrm{uAGT} / \mathrm{Cr})$, and $\ln (\mathrm{uR} / \mathrm{Cr})$ were associated with proteinuria decrement. Subsequent multivariable analysis identified baseline $\ln (\mathrm{uAGT} / \mathrm{Cr})$ as an independent predictor of proteinuria decrement (OR 1.372 , 95\% CI, 1.068-1.762, $P=0.013$ ).
Subgroup analysis regarding long-term renal outcome was performed in 56 patients who agreed to provide the information and were followed-up for 5 years after the end of study period. None of the patients progressed to ESRD. The characteristics and urinary AGT and renin profiles of these patients were summarized supplementary Table 1. Mean arterial pressure at 5 years was not different between the patients with uPCR $<1 \mathrm{mg} / \mathrm{mg}$ and $1 \geq \mathrm{mg} /$ $\mathrm{mg}$ at 5 years. The eGFR at 5 years was higher in patients with $\mathrm{UPCR}<1 \mathrm{mg} / \mathrm{mg}$ compared with those with uPCR $1 \geq \mathrm{mg} / \mathrm{mg}$ at 5 years $(61.8 \pm 18.9$ vs $45.0 \pm 21.6 \mathrm{~mL} / \mathrm{min} /$ $\left.1.73 \mathrm{~m}^{2}, P=0.003\right)$. The $\Delta \ln (\mathrm{uAGT} / \mathrm{Cr})$ at 24 weeks was an independent predictor for $\mathrm{uPCR}<1 \mathrm{mg} / \mathrm{mg}$ at 5 years in the models adjusted for DM, hypertension, baseline eGFR, baseline uPCR, and $\triangle \mathrm{uPCR}$ at 24 weeks (Table 4 ). $\mathrm{DM}$ was the only predictive factor for a change in eGFR at 5 years (eGFR at 5 years minus baseline eGFR) $(\beta-$ 15.038, $P<0.001$ ) (supplementary Table 2).

\section{Discussion}

This study demonstrates that baseline urinary AGT excretion and changes in urinary AGT levels by ARBs have prognostic potential in predicting the antiproteinuric effects of ARBs in patients with overt proteinuria. Patients with higher baseline urinary AGT excretion showed significant antiproteinuric effects of ARBs. In addition, overt proteinuria disappeared during the 5 years of follow-up in patients with a significant decrease in urinary AGT after short-term (24 weeks) valsartan treatment. These longterm effects were independent of a decrease in proteinuria during the short-term valsartan treatment.

The antiproteinuric effects of ARBs were associated with baseline urinary AGT and urinary renin levels in our study. In our previous study including biopsy-proven glomerulonephritis patients, patients with high urinary AGT and

Table 2 Urinary AGT and renin levels and related factors according to the degree of proteinuria decrement at 24 weeks of valsartan treatment

\begin{tabular}{|c|c|c|c|}
\hline & $\begin{array}{l}\text { Non-decrement }{ }^{a} \\
(\boldsymbol{n}=152)\end{array}$ & $\begin{array}{l}\text { Decrement }{ }^{\mathbf{b}} \\
(\boldsymbol{n}=53)\end{array}$ & $P$ \\
\hline Valsartan dose at 24 weeks & & & 0.668 \\
\hline $320 \mathrm{mg}$ & $96(63.2 \%)$ & $31(58.5 \%)$ & \\
\hline $160 \mathrm{mg}$ & $48(31.6 \%)$ & $18(34.0 \%)$ & \\
\hline $80 \mathrm{mg}$ & $8(5.3 \%)$ & $4(7.5 \%)$ & \\
\hline Baseline UPCR & $1.64(1.22-2.49)$ & $2.66(1.84-3.95)$ & $<0.001$ \\
\hline Baseline MAP & $111.4 \pm 12.0$ & $112.1 \pm 12.3$ & 0.713 \\
\hline Baseline In(uAGT/Cr) ( $\mu \mathrm{g} / \mathrm{g})$ & $3.29(2.53-4.21)$ & $3.92(3.39-4.59)$ & 0.004 \\
\hline Baseline In(uR/Cr) (pg/g) & $-1.25(-2.11-0.47)$ & $-0.35(-1.96-1.73)$ & 0.047 \\
\hline$\Delta \ln (\mathrm{uAGT} / \mathrm{Cr})(\mu \mathrm{g} / \mathrm{g})^{c}$ & $0.00(-0.79-0.63)$ & $-0.76(-2.07-0.80)$ & $<0.001$ \\
\hline
\end{tabular}

Abbreviations: MAP mean arterial pressure, $u A G T / C r$ urinary angiotensinogen/creatinine ratio, $u P C R$ urinary protein/creatinine ratio, $u R / C r$ urinary renin/creatinine ratio

${ }^{a}$ Non-decrement: patients with $\mathrm{UPCR}$ decrement $<1 \mathrm{mg} / \mathrm{mg}$

${ }^{b}$ Decrement: patients with uPCR decrement $\geq 1 \mathrm{mg} / \mathrm{mg}$

${ }^{\mathrm{c}} \Delta \ln (\mathrm{uAGT} / \mathrm{Cr})=[\ln (\mathrm{uAGT} / \mathrm{Cr})$ at 24 weeks] - [baseline $\ln (\mathrm{uAGT} / \mathrm{Cr})]$ 
Table 3 Predictive factors for proteinuria decrement ${ }^{\mathrm{a}}$ after 24 weeks of valsartan treatment

\begin{tabular}{|c|c|c|c|c|c|c|}
\hline & \multicolumn{3}{|c|}{ Univariable } & \multicolumn{3}{|c|}{ Multivariable } \\
\hline & $\mathrm{OR}$ & $95 \% \mathrm{Cl}$ & $P$ & $\mathrm{OR}$ & $95 \% \mathrm{Cl}$ & $P$ \\
\hline History of hypertension & 1.150 & $0.608-2.174$ & 0.667 & & & \\
\hline History of DM & 2.105 & $1.005-4.411$ & 0.049 & 2.091 & $0.964,4.536$ & 0.062 \\
\hline Previous RAS inhibitor use & 0.651 & $0.354-1161$ & 0.143 & & & \\
\hline MAP $(\mathrm{mmHg})$ & 1.007 & $0.981-1.003$ & 0.600 & & & \\
\hline $\mathrm{eGFR}\left(\mathrm{mL} / \mathrm{min} / 1.73 \mathrm{~m}^{2}\right)$ & 1.006 & & 0.256 & & & \\
\hline Baseline $\ln (\mathrm{uAGT} / \mathrm{Cr})(\mu \mathrm{g} / \mathrm{g})$ & 1.387 & $1.082-1778$ & 0.010 & 1.338 & $1.035,1.729$ & 0.026 \\
\hline Baseline $\ln (\mathrm{uR} / \mathrm{Cr})(\mathrm{pg} / \mathrm{g})$ & 1.199 & $1.022-1.407$ & 0.026 & 1.073 & $0.901,1.278$ & 0.430 \\
\hline$\Delta \mathrm{uNa} / \mathrm{Cr}(\mathrm{mmol} / \mathrm{mmol})^{\mathrm{b}}$ & 0.877 & $0.693-1.111$ & 0.227 & & & \\
\hline
\end{tabular}

Abbreviations: $\mathrm{Cl}$ confidence interval, DM diabetes mellitus, eGFR estimated glomerular filtration rate, MAP mean arterial pressure, $O R$ odds ratio, $R A S$ renin angiotensin system, $u A G T / C r$ urinary angiotensinogen/creatinine ratio, $u N a / C r$ urinary sodium/creatinine ratio, $u P C R$ urinary protein/creatinine ratio, $u R / C r$ urinary renin/creatinine ratio

Multivariable logistic regression analysis was conducted with variables with $P<0.1$ in the univariable models

aproteinuria decrement: decrease in $\mathrm{UPCR} \geq 1 \mathrm{mg} / \mathrm{mg}$

${ }^{\mathrm{b}} \triangle \mathrm{uNa} / \mathrm{Cr}=[\mathrm{uNa} / \mathrm{Cr}$ at 24 weeks] - [baseline $\mathrm{uNa} / \mathrm{Cr}]$

renin showed significantly decreased proteinuria and increased eGFR during RAS-inhibitor treatment [8]. However, another study of patients with non-diabetic kidney disease with substantial proteinuria reported that the percent change in urinary AGT, not baseline urinary AGT, was associated with the percentage change in proteinuria during losartan treatment [16]. These conflicting results from previous studies may be caused from differences in underlying kidney disease, the small number of patients, and the short follow-up period. In patients with the same extent of proteinuria, the intrarenal RAS activity and the response to RAS-inhibitors may vary depending on the type of kidney disease. This study included a relatively large number of patients with diverse proteinuric kidney diseases, including diabetic or hypertensive nephropathy and glomerulonephritis, and approximately $30 \%$ of patients were followed up for 5 years. Therefore, we believe that our results reflect overall clinical relevance of urinary AGT excretion during treatment with RAS inhibitors for overt proteinuria.

Consistent with previous reports, urinary AGT decreased during valsartan treatment in our study. Although one animal study reported that the primary source of renal angiotensin II is liver-derived AGT [23], other studies have demonstrated that AGT is mainly produced in the proximal tubules and secreted into the tubular lumen $[24,25]$. Plasma AGT appears to be unfiltered in the glomeruli due to its molecular weight (52$64 \mathrm{kDa}$ ) and negative charge [26]. Urinary AGT excretion reflects intrarenal RAS activity rather than filtration of plasma AGT in humans, but those studies only included patients with minimal proteinuria $[9,27,28]$. Like albumin, if the integrity of the filtration barrier is damaged, a large amount of plasma AGT can be filtered and excreted into the urine $[8,23]$. Therefore, we cannot exclude the possibility that some portion of urinary AGT in patients with heavy proteinuria might be filtered from systemic AGT through a damaged filtration barrier. However, decreased urinary AGT excretion at 24 weeks was associated with antiproteinuric effects of valsartan up to 5 years later, independent of a decrease in proteinuria at 24 weeks, supporting the value of urinary AGT as a biomarker reflecting intrarenal RAS activity.

There was no correlation between urinary AGT or renin excretion and the change in eGFR at 24 weeks as well as at 5 years, although baseline urinary AGT and renin excretion were negatively correlated with baseline eGFR. Urinary AGT and renin excretion are negatively correlated with eGFR [21, 27, 29], but the relationship

Table 4 Predictive factors for uPCR $<1 \mathrm{mg} / \mathrm{mg}$ at 5 years posttreatment

\begin{tabular}{|c|c|c|c|c|c|c|c|c|c|}
\hline & \multicolumn{3}{|c|}{ Model $1^{\mathbf{a}}$} & \multicolumn{3}{|c|}{ Model $2^{\mathbf{b}}$} & \multicolumn{3}{|c|}{ Model $3^{c}$} \\
\hline & $\mathrm{OR}$ & $95 \% \mathrm{Cl}$ & $P$ & $\mathrm{OR}$ & $95 \% \mathrm{Cl}$ & $P$ & $\mathrm{OR}$ & $95 \% \mathrm{Cl}$ & $P$ \\
\hline $\ln (\mathrm{UAGT} / \mathrm{Cr})(\mu \mathrm{g} / \mathrm{g})$ & 0.971 & $0.654-1.441$ & 0.882 & 1.548 & $0.868-2.760$ & 0.139 & 0.898 & $0.586-1.374$ & 0.620 \\
\hline$\Delta \ln (u A G T / C r)(\mu g / g)^{d}$ & 0.361 & $0.195-0.668$ & 0.001 & 0.287 & $0.126-0.654$ & 0.003 & 0.379 & $0.201-0.715$ & 0.003 \\
\hline $\ln (u R / C r)(p g / g)$ & 0.945 & $0.652-1.363$ & 0.753 & 1.105 & $0.733-1.666$ & 0.633 & 1.020 & $0.691-1.505$ & 0.922 \\
\hline
\end{tabular}

Abbreviations: $\mathrm{Cl}$ confidence interval, $\mathrm{OR}$ odds ratio, $u A G T / \mathrm{Cr}$ urinary angiotensinogen/creatinine ratio, $u R / C r$ urinary renin/creatinine ratio

${ }^{a}$ Model 1: Adjusted for diabetes mellitus, hypertension, and baseline eGFR

${ }^{\mathrm{b}}$ Model 2: Adjusted for baseline UPCR, diabetes mellitus, hypertension, and baseline eGFR

${ }^{c}$ Model 3: Adjusted for $\triangle \mathrm{UPCR}$ at 24 weeks (UPCR at 24 weeks - baseline uPCR), diabetes mellitus, hypertension, and baseline eGFR

${ }^{\mathrm{d}} \Delta \ln (\mathrm{UAGT} / \mathrm{Cr})=[\ln (\mathrm{uAGT} / \mathrm{Cr})$ at 24 weeks] - [baseline $\ln (\mathrm{uAGT} / \mathrm{Cr})]$ 
between urinary AGT or renin excretion and changes in renal function has not been well studied. Previous studies have suggested that baseline AGT excretion may have limited value as a prognostic factor in predicting changes in renal function during RAS-inhibitor treatment, although it has a negative correlation with changes in renal function without RAS inhibitors [8, 27, 30, 31]. RAS inhibitors seem to attenuate the differences in renal function changes between patients with high and low urinary AGT by inhibiting intrarenal RAS and subsequently decreasing proteinuria. However, a large-scale study with a long-term follow-up period is needed to clarify this issue.

Previous studies have suggested that urinary renin may be a better marker of intrarenal RAS activity than urinary AGT, especially in patients with heavy proteinuria [18]. However, our study showed that urinary renin excretion was not superior to urinary AGT for predicting the antiproteinuric effects of ARBs. The values of urinary renin excretion were relatively lower and showed a more skewed distribution compared to urinary AGT even though a previously verified method for measuring urinary renin was used [8]. Since a significant number of patients showed very low urinary renin levels close to the detection limit at the baseline, we did not measure urinary renin at 24 weeks. Urinary AGT seems to be a better biomarker for evaluating intrarenal RAS activity based on the current methodological limitations of urinary renin measurement.

There were several limitations in this study. First, our study included heterogeneous kidney diseases because overt proteinuria was the main inclusion criterion. The response to RAS inhibitors can be affected by the underlying kidney disease, and further studies enrolling a more homogenous population are required. However, our study has clinical relevance as it shows the overall relevance of urinary AGT or renin in patients with overt proteinuria receiving $A R B$ treatment. Second, a substantial number of patients were excluded due to insufficient data and a relatively small portion of patients was followed up for 5 years. Although these issues might cause selection bias, our study has a relatively large number of patients with overt proteinuria and the longest follow-up period compared with many previous studies. Third, the methodological limitation in measuring urinary renin activity also hindered the analysis although we used a previously verified method. The lack of serial follow-up data of urinary renin at 8 and 24 weeks after starting valsartan may limit generalization of our study results. Further studies using a more sensitive and precise method in a larger number of patients are required to investigate the clinical predictive usefulness of urinary renin levels in patients with overt proteinuria.

\section{Conclusions}

Our study demonstrated the clinical relevance of urinary AGT as a biomarker for predicting antiproteinuric effects of RAS inhibitors in both short-term and long-term follow-up periods. Both baseline urinary AGT excretion and urinary AGT changes during a short-term follow-up period may be used as potential surrogate markers for the effectiveness of RAS inhibitor treatment for patients with overt proteinuria.

\section{Supplementary information}

Supplementary information accompanies this paper at https://doi.org/10. 1186/s12882-020-01825-6.

Additional file 1: Supplementary table 1. Characteristics and urinary

AGT and renin of 56 patients who were followed up to 5 years.

Supplementary table 2 . Predictive factors for change in $\mathrm{GFR}^{\mathrm{a}}$ from baseline to 5 years in 56 patients.

\section{Abbreviations}

AGT: Angiotensinogen; ARB: Angiotensin receptor blocker; CKD: Chronic kidney disease; UAGT/Cr: Urinary angiotensinogen/creatinine ratio; eGFR: Estimated glomerular filtration rate; RAS: Renin angiotensin system; $\mathrm{UNa} / \mathrm{Cr}$ : Urinary sodium/creatinine ratio; UPCR: Urinary protein/creatinine ratio; uR/Cr: Urinary renin/creatinine ratio

\section{Acknowledgements}

The authors sincerely thank Kyung Hee Choi, Ji Woo Kim, and Woo Hyun Lee of the Samsung Bioresearch Institute (Seoul, Korea) for their technical assistance. We also thank Suhnggwon Kim (Department of Nephrology, College of Medicine, Seoul National University, Seoul, Republic of Korea), Kyu-Beck Lee (Division of Nephrology, Department of Internal Medicine, Kangbuk Samsung Hospital, Sungkyunkwan University School of Medicine, Seoul, Republic of Korea), Min Ok Kim (Division of Nephrology, Eulji University Hospital, Eulji University College of Medicine, Daejeon, Republic of Korea), Young-Ki Lee (Division of Nephrology, Department of Internal Medicine, Hallym University College of Medicine, Seoul, Republic of Korea), Sung Gyun Kim (Department of Internal Medicine, Kidney Research Institute, Hallym University Sacred Heart Hospital, Anyang, Republic of Korea), Jun Young Do (Division of Nephrology, Department of Internal Medicine, Yeungnam University Hospital, Daegu, Republic of Korea), Ho Jun Chin (Division of Nephrology, Department of Internal Medicine, Seoul National University Bundang Hospital, Seoul, Republic of Korea), Dong-Jin Oh (Division of Nephrology, Department of Internal Medicine, Chung-Ang University Hospital, Chung-Ang University College of Medicine, Seoul, Republic of Korea), and Young Joo Kwon (Nephrology Research Institution, Korea University Guro Hospital, Seoul, Republic of Korea) for their contribution to enrollment of patients.

\section{Authors' contributions}

H.R.J. and Y.G.K. developed research idea and study design. J.S.J and D.H.K. performed data acquisition. J.S.J, D.H.K., H.R.J., J.E.L., W.S.H., H.Y.K., D.J.K. and Y.G.K. contributed to data analysis and interpretation. H.R.J., J.E.L., W.S.H., H.Y.K., D.J.K. and Y.G.K. provided conceptual support and advice. J.S.J., D.H.K., and H.R.J. performed statistical analysis and drafting manuscript. J.S.J, D.H.K, and H.R.J. take responsibility for the integrity of the data analysis. All the authors have accepted responsibility for the entire content of this submitted manuscript and approved submission.

\section{Funding}

This study was supported by the Korean Society of Nephrology. The funding organization played no role in the study design; in the collection, analysis, and interpretation of data; in the writing of the report; or in the decision to submit the report for publication.

\section{Availability of data and materials}

The datasets used and/or analyses during the current study are available from the corresponding author on reasonable request. The requirement should first be submitted to ethical committee. 


\section{Ethics approval and consent to participate}

The Institutional Review Board (IRB) of each institution (Chung-Ang University Hospital, Chungbuk National University Hospital, Eulji University Hospital, Hallym University Kangnam Sacred Heart Hospital, Hallym University Sacred Heart Hospital, Kangbuk Samsung Hospital, Korea University Guro Hospital, Samsung Medical Center, Seoul National University Bundang Hospital, Seoul National University Hospital, Yeungnam University Hospital [in alphabetical order]) approved the protocol in compliance with the Declaration of Helsinki (representative institution: Samsung Medical Center, IRB number 2008-07-089). Written informed consent was obtained from all patients.

\section{Consent for publication}

Not applicable.

\section{Competing interests}

The authors declare that they have no competing interests.

\section{Author details}

'Division of Nephrology, Department of Medicine, Samsung Medical Center, Sungkyunkwan University School of Medicine, 81 Irwon-ro, Gangnam-gu, Seoul 06351, Republic of Korea. ${ }^{2}$ Division of Nephrology, Department of Medicine, Chungbuk National University Hospital, Cheongju 28644, Republic of Korea.

Received: 20 October 2019 Accepted: 22 April 2020

Published online: 01 May 2020

\section{References}

1. Bakris GL. Slowing nephropathy progression: focus on proteinuria reduction. Clin J Am Soc Nephrol. 2008;3(Suppl 1):S3-10.

2. Group G. Randomised placebo-controlled trial of effect of ramipril on decline in glomerular filtration rate and risk of terminal renal failure in proteinuric, non-diabetic nephropathy. Lancet. 1997;349:1857-63.

3. Kobori $\mathrm{H}$, Urushihara M. Augmented intrarenal and urinary angiotensinogen in hypertension and chronic kidney disease. Pflugers Arch. 2013;465:3-12.

4. Kobori H, Nishiyama A, Abe Y, Navar LG. Enhancement of intrarenal angiotensinogen in Dahl salt-sensitive rats on high salt diet. Hypertension. 2003:41:592-7.

5. Kobori H, Ozawa Y, Suzaki Y, Nishiyama A. Enhanced intrarenal angiotensinogen contributes to early renal injury in spontaneously hypertensive rats. J Am Soc Nephrol. 2005;16:2073-80.

6. Kobori H, Nishiyama A, Harrison-Bernard LM, Navar LG. Urinary angiotensinogen as an indicator of intrarenal angiotensin status in hypertension. Hypertension. 2003;41:42-9.

7. Jang HR, Kim SM, Lee YJ, Lee JE, Huh W, Kim DJ, et al. The origin and the clinical significance of urinary angiotensinogen in proteinuric $\lg A$ nephropathy patients. Ann Med. 2012:44:448-57.

8. Jang HR, Jeon J, Park JH, Lee JE, Huh W, Oh HY, et al. Clinical relevance of urinary angiotensinogen and renin as potential biomarkers in patients with overt proteinuria. Transl Res. 2014;164:400-10.

9. Kobori H, Alper AB Jr, Shenava R, Katsurada A, Saito T, Ohashi N, et al. Urinary angiotensinogen as a novel biomarker of the intrarenal reninangiotensin system status in hypertensive patients. Hypertension. 2009;53: 344-50.

10. Kim SM, Jang HR, Lee YJ, Lee JE, Huh WS, Kim DJ, et al. Urinary angiotensinogen levels reflect the severity of renal histopathology in patients with chronic kidney disease. Clin Nephrol. 2011;76:117-23.

11. Saito $T$, Urushihara $M$, Kotani $Y$, Kagami $S$, Kobori H. Increased urinary angiotensinogen is precedent to increased urinary albumin in patients with type 1 diabetes. Am J Med Sci. 2009;338:478-80.

12. Sarnak MJ, Astor BC. Implications of proteinuria: CKD progression and cardiovascular outcomes. Adv Chronic Kidney Dis. 2011;18:258-66.

13. Peterson JC, Adler S, Burkart JM, Greene T, Hebert LA, Hunsicker LG, et al. Blood pressure control, proteinuria, and the progression of renal disease. The modification of diet in renal disease study. Ann Intern Med. 1995;123: 754-62.

14. Weinstock Brown W, Keane WF. Proteinuria and cardiovascular disease. Am J Kidney Dis. 2001;38:S8-s13.
15. Mills KT, Kobori H, Hamm LL, Alper AB, Khan IE, Rahman M, et al. Increased urinary excretion of angiotensinogen is associated with risk of chronic kidney disease. Nephrol Dial Transplant. 2012;27:3176-81.

16. Lee YJ, Cho S, Kim SR, Jang HR, Lee JE, Huh W, et al. Effect of losartan on proteinuria and urinary angiotensinogen excretion in non-diabetic patients with chronic kidney disease. Postgrad Med J. 2011;87:664-9.

17. Zhuang Z, Bai Q, AL, Liang Y, Zheng D, Wang Y. Increased urinary angiotensinogen precedes the onset of albuminuria in normotensive type 2 diabetic patients. Int J Clin Exp Pathol. 2015;8:11464-9.

18. van den Heuvel M, Batenburg WW, Jainandunsing S, Garrelds IM, van Gool $J M$, Feelders RA, et al. Urinary renin, but not angiotensinogen or aldosterone, reflects the renal renin-angiotensin-aldosterone system activity and the efficacy of renin-angiotensin-aldosterone system blockade in the kidney. J Hypertens. 2011;29:2147-55.

19. Levey AS, Coresh J, Greene T, Stevens LA, Zhang YL, Hendriksen S, et al. Using standardized serum creatinine values in the modification of diet in renal disease study equation for estimating glomerular filtration rate. Ann Intern Med. 2006;145:247-54.

20. Jang HR, Lee YJ, Kim SR, Kim SG, Jang EH, Lee JE, et al. Potential role of urinary angiotensinogen in predicting antiproteinuric effects of angiotensin receptor blocker in non-diabetic chronic kidney disease patients: a preliminary report. Postgrad Med J. 2012;88:210-6.

21. Kobori H, Ohashi N, Katsurada A, Miyata K, Satou R, Saito T, et al. Urinary angiotensinogen as a potential biomarker of severity of chronic kidney diseases. J Am Soc Hypertens. 2008;2:349-54.

22. Krop M, Garrelds IM, de Bruin RJ, van Gool JM, Fisher ND, Hollenberg NK, et al. Aliskiren accumulates in renin secretory granules and binds plasma prorenin. Hypertension. 2008;52:1076-83.

23. Matsusaka T, Niimura F, Shimizu A, Pastan I, Saito A, Kobori H, et al. Liver angiotensinogen is the primary source of renal angiotensin II. J Am Soc Nephrol. 2012;23:1181-9.

24. Nakano D, Kobori H, Burford JL, Gevorgyan H, Seidel S, Hitomi H, et al. Multiphoton imaging of the glomerular permeability of angiotensinogen. J Am Soc Nephrol. 2012;23:1847-56.

25. Rohrwasser A, Morgan T, Dillon HF, Zhao L, Callaway CW, Hillas E, et al. Elements of a paracrine tubular renin-angiotensin system along the entire nephron. Hypertension. 1999;34:1265-74.

26. Kobori H, Nangaku M, Navar LG, Nishiyama A. The intrarenal reninangiotensin system: from physiology to the pathobiology of hypertension and kidney disease. Pharmacol Rev. 2007;59:251-87.

27. Yamamoto T, Nakagawa T, Suzuki H, Ohashi N, Fukasawa H, Fujigaki Y, et al. Urinary angiotensinogen as a marker of intrarenal angiotensin II activity associated with deterioration of renal function in patients with chronic kidney disease. J Am Soc Nephrol. 2007;18:1558-65.

28. Urushihara M, Kondo S, Kagami S, Kobori H. Urinary angiotensinogen accurately reflects intrarenal renin-angiotensin system activity. Am J Nephrol. 2010;31:318-25.

29. Juretzko A, Steinbach A, Hannemann A, Endlich K, Endlich N, Friedrich N, et al. Urinary angiotensinogen and renin excretion are associated with chronic kidney disease. Kidney Blood Press Res. 2017;42:145-55.

30. Lee MJ, Kim SS, Kim IJ, Song SH, Kim EH, Seo JY, et al. Changes in urinary angiotensinogen associated with deterioration of kidney function in patients with type 2 diabetes mellitus. J Korean Med Sci. 2017;32:782-8.

31. Satirapoj B, Aramsaowapak K, Tangwonglert T, Supasyndh O. Novel tubular biomarkers predict renal progression in type 2 diabetes mellitus: a prospective cohort study. J Diabetes Res. 2016;2016:3102962.

\section{Publisher's Note}

Springer Nature remains neutral with regard to jurisdictional claims in published maps and institutional affiliations. 